\title{
La universidad intercultural Amawtay Wasi del Ecuador, un proyecto atrapado en la colonialidad del poder
}

Amawtay Wasi Intercultural University of Ecuador, a trapped project in the coloniality of power

Luis Fernando Sarango Macas ${ }^{1}$

\section{Resumen}

En la década de los noventa del siglo XX, gran parte de los pueblos originarios de Abya Yala², demandan ante sus Estados la creación de un espacio de educación superior propio que puede llamarse Universidad. En el Ecuador, se logra la creación de la Universidad Intercultural de las Nacionalidades y Pueblos Indígenas "Amawtay Wasi", mediante ley de la república en 2004. Este es uno de los proyectos más emblemáticos del movimiento indígena ecuatoriano, cuyos principales promotores fueron la Confederación de Nacionalidades Indígenas del Ecuador (CONAIE) y el Instituto Científico de Culturas Indígenas (ICCI). Tras ocho años de sobrevivir a las tensiones creadas por un Estado colonial, mediante el argumento técnico-político llamado "Evaluación", se aplica el "Apartheid Epistémico" cerrando definitivamente la Universidad. A los cuatro años de resistencia ante el atropello y regresión de derechos, el presidente Lenin Moreno ofrece restituir el derecho a la Educación Superior. Efectivamente, en el contexto de un "diálogo" y la reforma de la LOES³, se recupera la Universidad ahora como "Publica Comunitaria", la misma que desde la conformación de la "Comisión Gestora" donde hay un solo indígena de sus siete miembros, como proyecto originalmente alternativo, se encuentra atrapado en los tentáculos de la "Colonialidad del Poder".

Palabras clave: Colonialidad de poder; moderno Estado Nación; apartheid epistémico; intercultural; universidad; diálogo de saberes.

\section{Abstract}

In the nineties of the last century, a large part of the original peoples of Abya Yala ${ }^{4}$ demand before their States the creation of their own higher education space that can be called University. In Ecuador, the creation of the Intercultural University of Indigenous Nationalities and Peoples "Amawtay Wasi" is achieved through the law of the republic in 2004. This is one of the most emblematic projects of the Ecuadorian indigenous movement, whose main promoters were the Confederation of Indigenous Nationalities of Ecuador (CONAIE) and the Scientific Institute of Indigenous Cultures (ICCI). After eight years of surviving the tensions created by a colonial state, through the technical-political argument called "Evaluation", the "Epistemic Apartheid" is applied, closing the University definitively. After four years of resistance to the abuse and regression of rights, President Lenin Moreno offers to restore the right to Higher Education.

\footnotetext{
1 Master en Docencia Universitaria. Pushak / Rector de la Pluriversidad Amawtay Wasi del Ecuador. Email: pushak1@yahoo.com DOI: https://orcid.org/00000003-0512-251X

2 Abya Yala, en lengua Guna. Abya: Sangre. Yala: Tierra. Tierra de Sangre. Si la sangre es la esencia de la vida, entonces será: Tierra de sangre vital o Tierra de Vida. Nombre que los pueblos originarios contemporáneos han dado al llamado continente americano.

3 Ley Orgánica de Educación Superior.

4 Abya Yala, in Guna language. Abya: Blood. Yala: Earth. Land of Blood If blood is the essence of life, then it will be: Land of vital blood or Land of Life. Name that contemporary indigenous peoples have given to the so-called American continent.
} 
Indeed, in the context of a "dialogue" and the reform of LOES5, the University is now recovering as a "Public Communitarian", the same as since the creation of the "Negotiation Commission" where there is only one indigenous member of its seven members, as an originally alternative project it is caught in the tentacles of the "Coloniality of Power".

Keywords: Coloniality of Power; Modern Nation State; epistemic apartheid; intercultural; University; Knowings dialogue.

\section{Introducción}

Uno de los proyectos más emblemáticos del movimiento indígena del Ecuador en los últimos tiempos ha sido el de la Universidad Intercultural de las Nacionalidades y Pueblos Indígenas "Amawtay Wasi" Debido a que se alcanzaban avances concretos en materia constitucional que dieron lugar a que en la constitución del 2008, el Ecuador sea declarado entre otros como estado Plurinacional e Intercultural7, casi estuvimos seguros de que un proyecto propio de Universidad podía ser una realidad. Es decir, que un estado plurinacional e intercultural constitucionalmente declarado ya podía tolerar, al menos, la existencia de un nuevo tipo de universidad, inspirada en lo diverso, en sus milenarias formas de hacer aprendizajes y en sus epistemes emergentes.

Nos equivocamos. El Estado plurinacional e intercultural constitucionalmente declarado, era solamente eso, declarado en el papel y con un largo camino por recorrer hasta que algún momento se haga realidad. Seguía siendo el típico Estado pro europeo, el Estado colonial, aquel Estado-Nación fundado unilateralmente por una minoría en 1830 y por lo tanto organizado en la idea de la existencia de razas superiores e inferiores, actualmente formando parte de las colonias sin colonias, bajo el paradigma civilizatorio occidental, conformando el capitalismo global, con el sueño inalcanzable de ser desarrollado. En resumen, un estado-nación funcionando desde la lógica de la "Colonialidad del Poder"

Dentro de esta lógica de Estado-Nación, constituía y constituye una verdadera utopía el funcionamiento de un nuevo tipo de universidad, surgida desde las necesidades originarias de hacer pensamiento y desarrollo de saberes con perspectiva intercultural. Es por ello que se explica su indiferencia al momento de los trámites de creación, su presionada aprobación, pero sin financiamiento, su tozudez por no permitir el funcionamiento desde los territorios usando tan solo un reglamento y frente a una sentencia favorable dictada por la Corte Constitucional. Por ello se explica entonces la aplicación del Apartheid Epistémico con la suspensión definitiva o cierre de la Universidad. Por ello se explica también, el supuesto salvataje de la universidad haciéndola estatal, pero para intervenirla, para controlarla, para atraparla en los tentáculos de la "Colonialidad del Poder".

En este breve ensayo, nos proponemos desempolvar la corta pero hermosa experiencia colectiva intercultural que nos permitió vivenciar el proyecto de universidad como tal. Queremos rememorar la

\footnotetext{
5 Organic Law of Higher Education.

6 Expresión en idioma Kichwa o Runa Shimi que en castellano quiere decir: Casa de la Sabiduría.

7 Ver el Art. 1 de la Constitución de la República del Ecuador del año 2008.

8 Teoria de análisis político e histórico formulada y usada por el pensador peruano Anibal Quijano. La colonialidad del poder es uno de los elementos constitutivos del patrón global de poder capitalista. Se funda en la imposición de una clasificación racial/étnica de la población del mundo como piedra angular de dicho patrón de poder, y opera en cada uno de los planos, ámbitos y dimensiones, materiales y subjetivas de la existencia cotidiana y a escala social. Se origina y mundializa a partir de América. Con la constitución de América, en el mismo momento y en el mismo movimiento histórico, el emergente poder capitalista se hace mundial, sus centros hegemónicos se localizan en las zonas situadas sobre el Atlántico -que después se identificarán como Europa-, y como ejes centrales de su nuevo patrón de dominación se establecen también la colonialidad y la modernidad. En otras palabras: con América el capitalismo se hace mundial, eurocentrado y la colonialidad y la modernidad se instalan, hasta hoy, como los ejes constitutivos de ese específico patrón de poder. (Quijano, 2007: 93-94).
} 
"Minka ${ }^{9}$ de Pensamiento", un ejercicio colectivo que nos ayudó aprender y a desaprender para recuperar la propuesta originaria de la Universidad que vivió y sobrevivió a los embates del Estado colonial con ocho años de actividad académica. Queríamos compartir con las nuevas generaciones que es natural para los Estados coloniales atrapar, inmovilizar y eliminar proyectos emanados desde las filosofías subalternas, desde las "razas" inferiores y desde cuando los vencedores se hicieron con el poder. Pero, también queríamos decirles que es posible una resistencia, una lucha con dignidad, una desobediencia epistémica que permita desenmascarar los viejos objetivos del Estado colonial y sus gobernantes de turno, pero también identificar a los "Felipillos"10, o los "Indígenas Funcionales ${ }^{11^{1}}$ al sistema imperante, que sin su vergonzosa labor, la concreción de nuestros derechos históricos no se verían postergados.

\section{Desarrollo}

\section{Una historia necesaria}

\section{La minka de pensamiento}

1996. Los entonces diputados de la república, compañeros Luis Macas y Leonidas Iza, conforman un equipo técnico integrado por indígenas y no indígenas con el objetivo principal de redactar un primer proyecto de Universidad Intercultural para las Nacionalidades y Pueblos Indígenas del Ecuador.

La propuesta escrita fue nada más y nada menos que la reproducción filosófica y estructural de una universidad convencional, de la universidad occidental, de aquellas vigentes en el Estado ecuatoriano desde la colonia. Así fue presentada originalmente a una Asamblea General de la Confederación de Nacionalidades Indígenas del Ecuador (CONAIE) y el Instituto Científico de Culturas Indígenas (ICCI) como entidades patrocinadoras.

El tipo de propuesta, causó muchas reacciones encontradas en los dirigentes de las organizaciones de base de las organizaciones regionales y nacional. El argumento de fondo consistía en que si bien ya existen demasiadas universidades desde el pensamiento occidental, qué ganaría el movimiento indígena creando una nueva universidad con la misma lógica y pensamiento occidental.

Desde esta polémica como premisa, los promotores de la creación de la universidad liderada por los compañeros Macas e Iza, planifican discutir este tema en sendos seminarios talleres a los cuales se les denominó "Minka de Pensamiento". A estos fueron invitados varios pensadores indígenas, no indígenas, líderes y lideresas, tanto a nivel nacional como internacional.

Los invitados a participar en esta minka debieron encontrar respuestas a interrogantes claves que saltaron a la mesa de discusiones y estas, entre otras, fueron:

1.- Según el pensamiento occidental, para fundamentar la construcción de una universidad como mínimo debe existir un pensamiento "desarrollado", teorizado y por escrito. Si el pensamiento indígena al parecer carece de suficiente bibliografía y de connotados teóricos, ¿cómo se puede tener fundamentos que le permitan construir una universidad?

\footnotetext{
9 Institución originaria parecida al trabajo voluntario, pero que se realiza dentro del principio de la reciprocidad.

${ }^{10}$ Alude a Felipillo. Que, según varios cronistas, Felipillo (o Felipe) fue un traductor nativo tallan que acompañó a Francisco Pizarro y Diego de Almagro en sus varias expediciones al Perú durante la Conquista del Imperio Inca. Su nombre real es desconocido. Considerado traidor de los nativos americanos por haber prestado su ayuda a los conquistadores al traducir el quechua en español.

11 Se llaman "Indígenas Funcionales" aquellos indígenas que desarticulados de sus comunidades y organizaciones viven en la gran ciudad ocupando, si acaso consiguen, puestos burocráticos medios y a veces altos, pero que sirven y se acomodan a los intereses de todos los gobiernos de turno, independientemente de su ideología o tendencia política, para que estos puedan aparentar que hay la participación indígena en la gestión estatal.
} 
2.- El movimiento indígena ha planteado su inconformidad con el pensamiento occidental y es más, ha planteado una ruptura epistémica con el mismo para dar a conocer su propia cosmovisión como aporte a la humanidad. Si nos encontramos en una época donde se han terminado las utopías y los pueblos y nacionalidades indígenas apenas somos considerados como subculturas, ¿qué esperanzas tenemos de ser escuchados en el mundo?

Las respuestas fueron contundentes: A la primera interrogante, nuestros mayores especialmente, nos dijeron que los jóvenes como producto de una educación occidental, quieren cumplir al pie de la letra lo que ella dice. Por eso quieren teoría y por escrito, quieren bibliografía y connotados teóricos. Todos los pueblos del mundo poseen su teoría del conocimiento y por tanto sabiduría, tienen su propia cosmovisión y filosofía, poseen principios y categorías, sus propios sistemas de escritura o pueden ser como el caso nuestro "ágrafos" pero con leguaje simbólico.

A la segunda interrogante: Que somos pueblos originarios que preexistimos a la invasión de occidente y a la configuración de los Estados Nación. Como depositarios de diversas sabidurías originarias, es nuestro deber aportar a la humanidad con nuestras propuestas para construir una nueva sociedad, la sociedad intercultural. Por tanto:

La creación de la Universidad Intercultural significa la oportunidad de emprender un diálogo teórico práctico desde lo intercultural; requiere la construcción de nuevos marcos conceptuales, analíticos, teóricos, en los cuales se vayan generando nuevos conceptos, categorías y nociones, desde el marco de la interculturalidad y la comprensión de la alteridad. (UINPI AW, 2004, p. 164).

Como primer producto de la "Minka de Pensamiento", construimos un modelo educativo que fue editado y publicado con el auspicio de UNESCO Ecuador, (2004), en tres idiomas: kichwa, español e inglés. El libro se llama: Sumak Yachaypi, Alli Kawsaypipash Yachakuna. Así, este se constituyó en nuestra propuesta filosófica y pedagógica de la Universidad, que en fin de cuentas es y será el referente que señala nuestro horizonte.

\section{La aprobación de la ley y el inicio de actividades}

Tomando en cuenta que a partir de 1996, se dio inicio a la tarea de preparar los requisitos y gestionar la creación de la universidad, para el 2003 se cumplían ya siete largos años.

Habíamos presentado en varias oportunidades la propuesta de creación con todos los requisitos y cuando se nos pedían que completáramos documentación, habíamos completado. Mas, se daba largas al asunto argumentando que no cumplíamos con todos los requisitos. Siempre había algún pretexto para que nuestra petición duerma el sueño de los justos. Para inicios del 2003, inclusive habíamos pasado con éxito el proceso de peritaje realizado por la Universidad Central del Ecuador y la Universidad de Cuenca. Para nosotros, todo estuvo en regla, excepto lo que nos fue imposible, obtener una partida presupuestaria en el Ministerio de Economía y Finanzas para que la universidad sea pública. Para lograr dicha partida, se necesitaba tener influencia política al muy alto nivel del gobierno, pero eso, en ese momento, no lo teníamos.

Ante esta situación, las entidades patrocinadoras CONAIE e ICCI, debieron tomar una decisión. Conscientes que sin la partida presupuestaria es imposible la aprobación de una universidad pública, pensaron que lo mejor sería entonces, adoptar la figura de particular cofinanciada por el Estado, toda vez que este tipo de reconocimiento hace posible contar con un apoyo económico puntual para la universidad. Pronto se nos hizo conocer que la figura de particular cofinanciada por el Estado ya no estaba vigente, quedando entonces la única alternativa, la figura de particular autofinanciada. 
Con estos antecedentes, las organizaciones provinciales, regionales y nacional, se dieron cita en la ciudad de Manta, provincia de Manabí, sitio en el que el Consejo Nacional de Educación Superior (CONESUP) en pleno, celebraría una reunión de rutina. El pleno del CONESUP, prácticamente sorprendido ante la presencia de cientos de indígenas de todo el país que pidieron ser recibidos en comisión general, el 26 de noviembre del 2003, por la noche, terminó aprobando el informe favorable obligatorio contenido en la Resolución RCP.S19.No.493.03 del 26 de noviembre del 2003.

A continuación, tras el trámite de rigor y un cabildeo político realizado por parte de los diputados del Movimiento Pachakutik ${ }^{12}$, el Congreso Nacional expide la Ley No. 2004-40 ${ }^{13}$, el mismo que fuera publicado en el Registro Oficial No. 393 del 5 de agosto del 2004, mediante la cual se crea oficialmente la Universidad Intercultural de las Nacionalidades y Pueblos Indígenas "Amawtay Wasi”.

El 30 de noviembre del 2005, el pleno del CONESUP, en sesión realizada en la ciudad de Ambato, aprueba sin observación alguna el Estatuto Orgánico de la Universidad Intercultural "Amawtay Wasi", con lo cual la universidad pasa a formar parte del Sistema Nacional de Educación Superior. En diciembre de ese mismo año, se matriculan en la licenciatura de Ciencias de la Educación con mención en Pedagogía Intercultural, cuarenta estudiantes saraguros y sesenta estudiantes shuar, dando un total de cien estudiantes que deben asistir a clases presenciales en la ciudad de Quito, Chakra "Conocoto". De esta forma, la Universidad inicia formalmente sus actividades académicas.

\section{Los ocho años de sobrevivencia}

Del 30 de noviembre del 2005, fecha de aprobación del estatuto orgánico y del inicio de las actividades académicas, al 4 de noviembre del 2013 se cuentan prácticamente ocho años de vida de la Universidad. Este lapso de ocho años de vida de la Universidad Amawtay Wasi, tiene un gran significado para el proyecto original, tomando en cuenta que todo este período se caracteriza por un clima de tensiones permanentes entre el Estado y la universidad. Para la Universidad es un período de sobrevivencia.

Una universidad sin recursos económicos por su condición de particular, con un estado que hace las veces de gendarme, que mediante un reglamento impide el funcionamiento autónomo especialmente en su estructura institucional territorializada, debió darse modos para cumplir con sus tres funciones sustantivas: docencia, investigación y extensión ${ }^{14}$.

En diciembre del 2005, se inicia la actividad académica con 100 estudiantes originarios que ingresan a la carrera o camino de Pedagogía Intercultural, pero lo más curioso es que dichos estudiantes provenían de los pueblos quizá más alejados con respecto a Quito, los saraguros situados en la provincia Loja, provincia fronteriza con el Perú y los shuar situados en la provincia de Morona Santiago, cuyos estudiantes venían inclusive desde la cordillera del Cutucú, selva amazónica fronteriza también con el Perú. Ya en el transcurso del 2006, en la misma ciudad de Quito, por así disponer la Ley de Educación Superior y su reglamento, se aperturaron los caminos o carreras de Arquitectura Ancestral y Agroecología.

La investigación, que generalmente se conocía como un área de y para especialistas, se logra transversalizar en la "estructura curricular" 15 y es una de las actividades permanentes en el objetivo de acortar la

\footnotetext{
12 El Movimiento Pachakutik, es un movimiento político electoral que en el Ecuador es conocido como el brazo político de la Confederación de Nacionalidades Indígenas del Ecuador CONAIE.

13 Esta es la Ley de creación de la Universidad Intercultural Amawtay Wasi, cuya virtud consiste en que en su Art. 4, reconoce una estructura chakanística.

${ }^{14}$ En el Ecuador a la extensión le llaman Vinculación con la sociedad.

${ }^{15}$ A la estructura curricular, en el modelo Amawtay Wasi, se le denomina "Chakana Curricular".
} 
brecha entre la teoría y la práctica. Así la investigación con su método "Vivencial Simbólico Relacional”", juega un papel preponderante en el eje de los Emprendimientos Comunitarios.

Es importante señalar que la sistematización y elaboración de "Módulos Informativos"17 o teóricos en temas de Saberes Originarios, forman parte de los procesos de investigación de parte de los docentes y de las comunidades. Todas estas actividades fueron realizables gracias a un Modelo Educativo propio de la Universidad llamado el “Kapak Ñan Pedagógico - Filosófico de la Universidad Intercultural Amawtay Wasi”.

En el campo de la extensión, denominado por el estado como vinculación con la sociedad, en los procesos de evaluación al que se sometió la universidad, se trató de explicar que una forma primordial de hacer extensión/vinculación, en nuestro caso, era precisamente hacer actividad académica desde los territorios de nuestras comunidades y pueblos. Pretendimos hacerles entender que los desvinculados de nuestra realidad eran las universidades convencionales y los evaluadores. Sin embargo, la obediencia epistémica a occidente hizo que se negaran a entender nuestra lógica, porque para ellos lo normal es que la universidad funcione con una matriz centralizada en una ciudad ${ }^{18} \mathrm{y}$ a partir de esa matriz se crean las extensiones, con una organización piramidal, de arriba hacia abajo y del centro hacia afuera, a la inversa de nuestra lógica.

A propósito de esta lucha por hacer comprender a un Estado colonial que el modelo institucional de nuestra universidad surgía desde los territorios de los pueblos originarios y no al revés, ante la tozudez de imponernos su razón centralista con un simple reglamento frente a disposiciones constitucionales como los Derechos colectivos del Art. 57 de la Constitución, el Convenio No. 179 de la OIT, así como la Declaración de la ONU, sobre los derechos de los pueblos Indígenas, frente a un cerco intencional para asfixiarnos a todas luces, en el mes de febrero del 2008, se interpuso una demanda por incumplimiento en contra del entonces CONESUP, ante la Corte Constitucional, obteniendo una sentencia favorable ${ }^{19}$ aunque no tan contundente como hubiéramos querido.

Esta sentencia, en lo fundamental declara el incumplimiento por parte del CONESUP, del Art. 4 de la Ley de Creación de la Universidad y el Art. 31 del Estatuto Orgánico, y en su parte resolutiva, además dispone:

4. En concordancia con el Convenio 169 de la OIT (artículos 2, 3, 4, 5, y 27), el CONESUP, se sujetará para la autorización solicitada por la Universidad Intercultural de las Nacionalidades y Pueblos Indígenas "AMAWTAY WASI", a las disposiciones de dicho convenio, en lo siguiente: a) la apertura de Programas Académicos en los territorios de las Nacionalidades y Pueblos Indígenas, según su cultura y cosmovisión; b) que en pleno ejercicio de la autonomía universitaria desarrolle sus propias modalidades y ponga en práctica sus propios métodos de aprendizaje y esto sirva como un aporte innovador de las Nacionalidades y Pueblos Indígenas al sistema nacional de Educación Superior; y, c) que implemente sus propios métodos de aprendizaje, sus modalidades, planes o programas que sean necesarios acordes con sus Centros del Saber y de conformidad con su Ley de creación, en estricto respeto a los derechos de las Nacionalidades y Pueblos Indígenas reconocidos en la Constitución de la República (Corte Constitucional; 2009, p. 28-29).

\footnotetext{
${ }^{16}$ El Método Vivencial Simbólico Relacional, es un método de aprendizaje e investigación que permitió el desarrollo del modelo Amawtay Wasi. Más tarde, este método, conjuntamente con los métodos usados por la Universidad Autónoma Indígena Intercultural UAIIN - CRIC de Colombia y la Universidad de las Regiones Autónomas de la Costa Caribe Nicaragüense URACCAN de Nicaragua, formarán el conjunto de metodologías de investigación propia llamada "Cultivo y Crianza de Sabidurías y Conocimientos CCRISAC".

17 Los Módulos Informativos, conjuntamente con la Investigación, los Emprendimientos Comunitarios y el Conversatorio son las cuatro actividades para generar aprendizaje.

18 "Civilización".

${ }^{19}$ Nos referimos a la Sentencia No. 0008-09-SAN-CC, caso No. 0027-09-AN, de fecha 9 de diciembre del 2009 dictada por la Corte Constitucional del Ecuador para el periodo de transición.
} 
A nivel internacional, en todo el continente americano o Abya Yala, la Universidad Intercultural Amawtay Wasi, ha sido un referente que ha incidido en todo este movimiento de los pueblos originarios que reivindica el derecho a tener una universidad desde, con y para dichos pueblos. Así, por ejemplo, ha servido de inspiración para que en Bolivia se creen tres universidades como la Universidad Aymara "Tupak Katari", la Universidad Quechua "Casimiro Huanca" y la Universidad Guaraní y pueblos de tierras Bajas "Apiaguaiki Tupa".

En los días 19 y 20 de abril del 2008, en la ciudad de Quito, las Universidades Amawtay Wasi como anfitriona, la Universidad Autónoma Indígena Intercultural (UAIIN-CRIC) de Colombia y la Universidad de las Regiones Autónomas de la Costa Caribe Nicaragüense (URACCAN), formalmente crean la Red de Universidades Indígenas, Interculturales y Comunitarias de Abya Yala (RUIICAY), con siete acuerdos fundacionales, de los cuales el acuerdo cinco, dice:

Aunar esfuerzos y voluntades políticas para exigir a los Estados el reconocimiento pleno y con apoyo financiero a nuestro derecho de contar y organizar instituciones de Educación Superior propias, conforme al Convenio 169 de la OIT, la Declaración de la ONU, sobre los Derechos de los Pueblos Indígenas, y a la luz de los Derechos Humanos. (RUIICAY, 2018, p. 12).

A partir del 2005 y hasta el 2013, la Universidad Amawtay Wasi, forma parte de la Red Universidad Indígena Intercultural UII, del Fondo Indígena Latinoamericano y del Caribe FILAC ${ }^{20}$, presidiendo en los últimos años su Consejo Académico.

A pesar de un período de ocho años lleno de tensiones causados principalmente por el Estado colonial, de la total escases de recursos económicos, hasta la infaltable oposición de una minoría de indígenas, se publicaron al menos 10 libros relacionados con la interculturalidad, la lengua Pukina y la arquitectura dentro de la línea editorial Amawta Runakunapak Yachay A.R.Y.

Se registraron legalmente cuatro carreras: 1.- Licenciatura en Ciencias de la Educación con mención en Pedagogía Intercultural; 2.- Ingeniería en Agroecología; 3.- Arquitectura con mención en Arquitectura Ancestral; y, Turismo Comunitario, este último que no llegó a ejecutarse. Para el 2013, año de la suspensión definitiva de la Universidad, graduamos a 232 estudiantes en los más diversos campos del nivel de Comunidades de Aprendizaje; 47 licenciados en Ciencias de la Educación con mención en Pedagogía Intercultural del nivel de Pregrado ${ }^{21} ; 31$ facilitadores en el Diplomado Superior en Investigación Intercultural nivel Postgrado; y 30 facilitadores en la Maestría en Derechos Humanos y Pueblos Indígenas nivel Postgrado. Quedaron listos para graduarse 10 estudiantes de agroecología y 5 estudiantes de arquitectura, así dos cohortes más de estudiantes en cada una de las tres carreras vigentes.

\section{El apartheid epistémico y la suspensión definitiva}

El Consejo de Evaluación, Acreditación y Aseguramiento de la Calidad de la Educación Superior (CEAACES), del Ecuador, en octubre del 2013, aplicó un proceso de "evaluación" a la Universidad Intercultural "Amawtay Wasi”. Esta "evaluación” constituyó un proceso burocrático necesario para poder argumentar técnicamente el cierre definitivo de la Universidad.

Como tenemos dicho, el objetivo primordial de esta llamada "evaluación" era, desaparecer del mapa académico a la Universidad "Amawtay Wasi”, principalmente por la intolerancia del pensamiento occidental

${ }^{20}$ El Fondo Indígena Latinoamericano y del Caribe FILAC, es un organismo internacional que tiene su sede en La Paz, Bolivia.

${ }^{21}$ Los títulos que se otorgaron fueron impresos en lengua materna del graduado y en castellano. 
colonialista del gobierno de Correa que se manifestó como un verdadero "Apartheid Epistémico"22 (Rabaka, 2010), contradiciendo inclusive el estatus jurídico actual de estado plurinacional que hoy ostenta constitucionalmente el Ecuador.

Sin tener la curiosidad al menos de preguntar por el "Modelo Educativo" llamado por nosotros "Camino Filosófico - Pedagógico" que anima la experiencia de la Universidad, pese a que lo hemos entregado en las manos del equipo evaluador el primer día que llegaron a la Administración Central en Quito y que no les interesaba si existiera o no, pues así se notó cuando dejaron abandonados los libros que contienen dicho modelo. Con el supuesto de que no existe tal Modelo Educativo de la Universidad Amawtay Wasi, aplicaron un aberrante "Modelo de Evaluación" que, se nota, proviene de un "modelo universal". Claro, en fin de cuentas, lo que interesaba era desaparecernos.

Miremos el Modelo de Evaluación elaborado unilateralmente por el CEAACES, y que nos fue impuesto invocando la LOES, una ley inferior respecto de la constitución y los tratados internacionales. La pregunta es. ¿Con este modelo de evaluación pertinente para "Universidades Occidentalizadas" (Grosfoguel, 2013), era posible evaluar un modelo educativo inspirado desde los pueblos originarios?

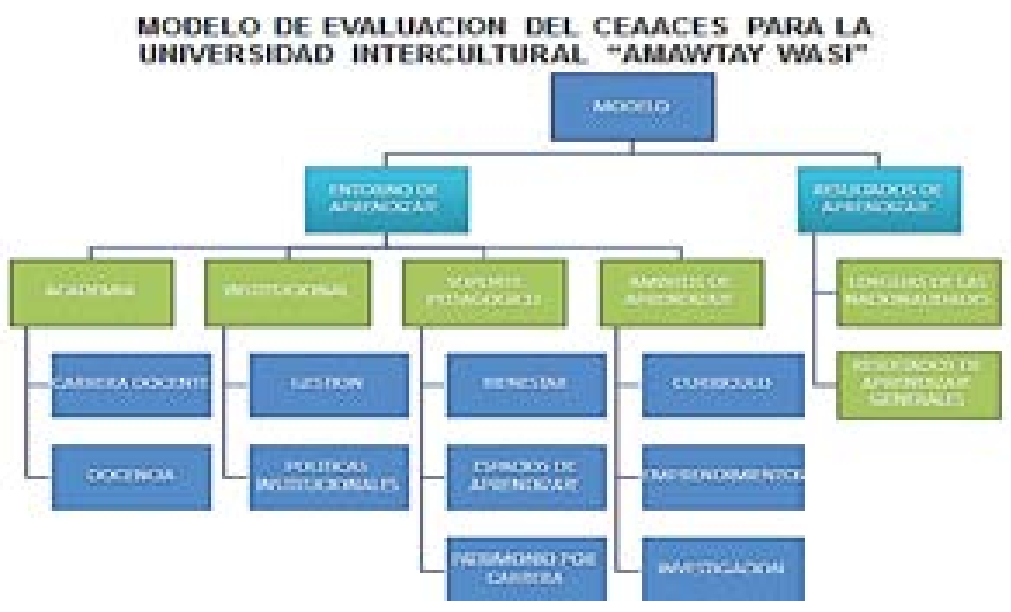

Fuente: CEAACES 2013.

Terminada una evaluación plagada de irregularidades, con grabaciones secretas, con el desprecio manifiesto al modelo educativo de la Universidad, con el olvido de llenar previamente las matrices de evaluación, el resultado nada sorprendente fue que habíamos obtenido la nota de 26,9 sobre cien, lo que dio como consecuencia que se resuelva la suspensión definitiva o cierre de la Universidad.

En efecto, mediante Resolución RPC-SE-12-No. 049-2013 del 4 de noviembre del 2013, el Consejo de Educación Superior (CES), resuelve: “... aprobar la suspensión definitiva de la Universidad Intercultural de las Nacionalidades y Pueblos Indígenas "Amawtay Wasi” y nombrar como Administrador Temporal al Dr. Luis Alfonso Álvarez Rodas" (CES, 2013, p. 5), quedando así cumplido el sueño tan esperado por Correa, suspender o mejor dicho, cerrar de manera definitiva la Universidad surgida desde la inspiración filosófica de los pueblos originarios, gestionada desde sus territorios y proyectada para la construcción del Estado plurinacional.

\footnotetext{
22 Originalmente, este término ha sido usado por el profesor Reiland Rabaka especialmente en su libro: Against Epistemic Apartheid (2010). Posteriormente, varios académicos latinoamericanos usaron este nombre como calificativo al acto de suspensión definitiva o cierre de la Universidad Intercultural Amawtay Wasi por parte del gobierno de Correa.
} 


\section{La etapa de resistencia}

Unas veinticinco personas ente directivos, técnicos y docentes de la Universidad cerrada, decidimos no aceptar la oferta del Estado, el de ser cooptados y muy bien pagados por el Estado para ser facilitadores o docentes en el "Plan de contingencia" ${ }^{23}$ que la SENESCYT ${ }^{24}$ y el CES, ya lo tenían preparado con anticipación. Claro está que varios de nuestros compañeros y compañeras, como no podía ser de otra manera, se fueron felices con el Estado, en fin de cuentas lo que les fue más importante era el dinero.

Para diciembre del 2013, los que tuvimos ante todo dignidad, decidimos constituirnos en una organización de resistencia epistémica, que nos permitiera denunciar ante el mundo los atropellos a los Derechos Humanos del que fuimos víctimas, pero además dar continuidad a nuestro pensamiento mediante la sistematización y difusión de nuestros saberes usando metodologías de investigación propias. Esta organización la denominamos "Pluriversidad Amawtay Wasi" ${ }^{25}$ que obtuvo su reconocimiento legal por parte del estado.

En el mes de noviembre de los años 2014 al 2017 organizamos eventos académicos con la participación de pensadores indígenas y no indígenas mediante los cuales convocábamos a reflexionar sobre el papel que juegan los estados coloniales y su "Colonialidad del Poder" en su proyecto irrenunciable de homogenización y modernización del capitalismo. Encontrábamos un buen espacio para reflexionar sobre el "Apartheid Epistémico" que fue practicado con el cierre de la Universidad y las luchas que debemos dar en el tiempo, debido a que nuestros derechos son irrenunciables.

Es importante señalar que con el apoyo de los entonces presidentes del ECUARUNARI ${ }^{26}$, Delfín Tenesaca y Yaku Pérez en su orden, adicionalmente presentamos una denuncia, respecto del cierre de la Universidad, ante la Comisión Interamericana de Derechos Humanos CIDH, en Washington.

\section{A los cuatro años del cierre renace la esperanza de recrear la universidad}

En las elecciones generales celebradas el 19 de febrero del 2017, Lenín Moreno, exvicepresidente, heredero político de Correa y candidato oficialista, alcanza un estrecho triunfo electoral. El 24 de mayo del 2017, se posesiona como presidente constitucional del Ecuador.

Una vez en el palacio presidencial, en la etapa inicial o de romance político, cuando todo presidente trata de aparentar que está dispuesto hacer todo lo que no hicieron sus antecesores, tratando de consolidar una nueva base social para su gobierno, invita por varias ocasiones a la dirigencia de la CONAIE para "dialogar" de manera pública en el palacio de Carondelet ${ }^{27}$. Desde luego que la dirigencia asiste a dicho diálogo por varias ocasiones. Las más sobresalientes 4 de julio y 11 de septiembre del 2017. En las dos reuniones y de manera pública, el presidente dijo: “... Que se devolverá la Educación Intercultural Bilingüe y la Universidad Indígena ... a sus legítimos dueños, es decir, a los pueblos y nacionalidades”.

Para el caso concreto de la Universidad Amawtay Wasi, cuya ley de creación se encontraba aún vigente y su espacio de resistencia la Pluriverisdad Amawtay Wasi se encontraba en pie de lucha, a los cuatro

\footnotetext{
${ }^{23}$ Este plan, consistía en graduar a todos los estudiantes de todas las carreras y niveles inferiores que aún no pudieron hacerlo antes del cierre de la Universidad. Para graduarlos había que hacer clases al clásico estilo de las universidades occidentalizadas, pidieron auspicio a la Universidad Central, Universidad de Cuenca y Universidad Andina para que acrediten los títulos a obtener. Trataron de insertar a los estudiantes en universidades convencionales, pero ante la oposición de estos, los cursos se culminaron con los planes de estudio de la Universidad Amawtay Wasi, pero a la manera occidental que resultaba más fácil.

24 Secretaria Nacional de Educación Superior, Ciencia, Tecnología e Innovación.

25 El término Pluriversidad, alude a la confluencia de varios universos de conocimientos y saberes. Es la diversidad de epistemologías que dialogan en pos de la vida.

${ }^{26}$ Confederación de Pueblos de la Nacionalidad Kichwa del Ecuador ECUARUNARI, organización regional que conforma la Confederación de Nacionalidades Indígenas del Ecuador CONAIE.

27 Se conoce con este nombre el palacio donde vive y tiene su despacho el Presidente de la República del Ecuador.
} 
años de suspensión o cierre de la Universidad, renacía la esperanza de recrearla, con toda su experiencia acumulada, con la totalidad de sus estudiantes graduados y esta vez "con recursos" dotados por el estado. Esa fue al menos, la ilusión inmediata que se nos presentaba.

En efecto, la CONAIE, en una Asamblea Nacional ampliada, con la presencia de las organizaciones regionales ECUARINARI y CONFENIAE ${ }^{28}$, conforma una Comisión Técnica de Educación que tendrá como objetivo sustentar y gestionar la restitución integral del Sistema de Educación Intercultural Bilingüe y dentro del cual funcione la Universidad desde nuestros territorios. Tratándose de Educación Superior se conforma una subcomisión que preside Luis Fernando Sarango, como ex rector de la Amawtay Wasi.

De inmediato se realizan gestiones y la SENESCYT, se muestra dispuesta a trabajar en la restitución de la Universidad. Se realizan reuniones técnicas y los ex directivos de la Universidad en su fase inicial exponen detalladamente los fundamentos filosóficos que dieron origen a la Universidad, las razones políticas y de discriminación que dieron como resultado el cierre de la Universidad y la propuesta para que se restituya la Universidad, pero esta vez con el carácter de "Pública Comunitaria". Inclusive se trabajó la conceptualización de lo "Publico Comunitario"29, que originalmente y como es costumbre, causó temor en el equipo jurídico de la SENESCYT.

Cuando el proyecto de Ley reformatoria a la LOES, estuvo casi listo desde la SENESCYT, los miembros de la Pluriversidad Amawtay Wasi, advirtieron al menos dos inconsistencias graves que pueden originar consecuencias negativas para la universidad restituida. La primera, es que prácticamente se elimina el Art. 4 de la Ley de creación, la misma que reconocía los cinco centros del saber o cinco espacios de la Chakana, que a la vez determinaba la estructura no occidental de la Universidad. La segunda, de manera innecesaria se dejaba intacta la disposición transitoria CUARTA de la Ley de creación, la misma que disponía la entrega de bienes por parte de los "patrocinadores originales" ${ }^{\circ}$. Al transformarse la universidad de particular a pública, esta disposición debió ser derogada y esto no sucedió. Lo que se supo luego, es que el grupo de "Indígenas Funcionales" encabezado por un funcionario del Ministerio del Interior, negociaba y acordaba la redacción final de la ley y luego organizaba reuniones con la Comisión de Educación para "formalizar" lo secretamente acordado.

El 12 de julio del 2018, la Asamblea Nacional aprueba la Ley Reformatoria a la Ley Orgánica de Educación Superior en cuya disposición reformatoria SEXTA, se encuentra la reforma a la Ley de creación de la Universidad del $2004^{31}$.

Tan pronto se inició la etapa de ejecución de la Ley reformada, se realizó un taller conjunto entre la Comisión de Educación de la CONAIE y el SENESCYT, para establecer una hoja de ruta en el cual y por su experiencia, varias comisiones lo lideraban y entre ellos ex técnicos de la Universidad Amawtay Wasi, etapa inicial. Esto incomodó a los indígenas funcionales que, con la complicidad de la SENESCYT liderada por Adrián Bonilla, reformularon la hoja de ruta y eliminaron de esta a todos los ex miembros de la Universidad, etapa inicial. Realizando así una limpieza total a fin de que sean los funcionales quienes lideren la "Restitución" de la Universidad Amawtay Wasi.

\footnotetext{
${ }^{28}$ Confederación de Nacionalidades de la Amazonía Ecuatoriana.

29 Entendido esto, - por un lado -, que es una institución creada bajo el amparo y para beneficio general del estado plurinacional, que es la expresión de la voluntad de todos quienes lo constituimos, y - por otro - que esta entidad tiene un carácter especial, porque se gestiona y se administra bajo el régimen comunitario, es decir, dentro del marco filosófico y jurídico legal de los derechos de los pueblos indígenas u originarios que vienen a ser la interpretación más próxima del ideal de vida comunitaria de estos pueblos.

${ }^{30}$ Los patrocinadores originales de la creación de la Universidad fueron: La Confederación de Nacionalidades Indígenas del Ecuador CONAIE y al Instituto Científico de Culturas Indígenas ICCI.

31 Esta Ley fue publicada en el Suplemento del Registro Oficial No. 297 del 2 de agosto del 2018.
} 
Así, para los "indígenas funcionales" al gobierno y el Estado colonial representado por la SENESCYT, sólo fuimos útiles hasta la promulgación de la Ley reformatoria a la LOES. Para lo posterior constituíamos un verdadero peligro.

\section{El proyecto de la Universidad Intercultural Amawtay Wasi, atrapado por la colonialidad del poder}

El proyecto originario de la Universidad Intercultural de las Nacionalidades y Pueblos Indígenas "Amawtay Wasi", en realidad era y es un proyecto transformador de la Educación Superior, que como hemos repetido por varias ocasiones, si bien surge desde los Pueblos y Nacionalidades, no es exclusivamente para ellos, sino que se proyecta para todas las sociedades como una alternativa a la hegemonía del orden epistémico mundial establecido.

Con una organización institucional chakanística de la Universidad lograda en la Ley 2004-40 del año 2004, la Universidad, planteaba desarrollar un sistema coordinado de educación superior desde los territorios de los Pueblos y Nacionalidades Indígenas a partir de sus propias teorías del conocimiento, que se derivó en acciones como:

1.- Hacer una educación superior desde, con y para los pueblos originarios.

2.- Hacer una educación superior con sus propias metodologías de aprendizaje e investigación.

3.- Hacer una educación superior, desde sus territorios y tomando a este como espacio propicio para recuperar, sistematizar y criar conocimientos.

4.- Hacer una educación superior descolonizadora.

Desde la misma recepción de la propuesta de creación de la Universidad, allá en el 2000, el Estado colonial ha adoptado su posición natural a su condición y esta es el de mirar con recelo y temor a que los "indios" tengan su propia universidad, y las consecuencias que puede tener el permitir que estos "indios" estudien y conozcan las realidades del mundo desde una visión multidimensional. Es decir, el Estado desde el primer día de gestión expresó su racismo, haciendo de este asunto tan simple, un problema político, jurídico y de "apartheid epistémico" 32 demasiado complicado.

Es por esta razón, que al momento de aparentar la restitución de la Universidad y de readecuar su funcionamiento, había que hacerlo con pinzas, había que tener cuidado en la expedición de la Ley cercenando artículos que corten las alas al proyecto originario. Desde luego, había que contar con los "indígenas funcionales" a la "Colonialidad del Poder", requisito clave para sus propósitos y que desde la propia colonia les ha dado buenos resultados.

Había que barrer con todos los que podían sostener el proyecto originario de la Universidad porque ciertamente estos constituyen un peligro para el orden establecido. Había que cuidar la conformación de la Comisión Gestora ${ }^{33}$, incluso violando su propio reglamento de conformación si fuera necesario como en efecto sucedió. Era necesario asegurarse que la universidad ahora en manos del Estado colonial no sea una universidad paria, que salga de los cánones establecidos por la convencionalidad, es decir que no sea una universidad que huela a indio.

\footnotetext{
32 Si los demandantes de la universidad hubieran sido empresarios o sacerdotes católicos, todo hubiera sido tan simple. Para los empresarios, el capitalismo es su mundo, para los sacerdotes les sobran convenios con la santa sede y desde la colonia fueron los encargados de la educación en América. Pero en este caso, son solamente "Indios".

${ }^{33}$ La comisión gestora de la Universidad la integran: Juan llicachi y Ángel Ramírez, representantes de la CONAIE; Ruth Moya y Rosa Endara, de la Academia; José Brito del Ministerio de Educación, Mateo Martínez por Senescyt, y como Secretaria Tania Molina. De todos ellos, solo Juan Ilicachi, es indígena Kichwa.
} 
De lo que se ha visto, los miembros de la Comisión Gestora han decidido partir de cero, es decir que para ellos no hubo una etapa previa de ocho años funcionando como universidad. Para la Comisión Gestora, la Universidad Intercultural Amawtay Wasi, existe solo a partir del 2 de agosto del 2018, fecha de publicación de la Ley reformatoria a la LOES. La negación y la invisibilización ha sido una práctica común del colonizador, pues se intenta anular y borrar todo signo de producción de conocimiento previo para poder imponer su proyecto educativo argumentando el vacío, el primitivismo, la barbarie de los antecesores para que lo "nuevo" tenga cabida e irremediable aceptación.

Por noticias publicadas en los medios de comunicación nacional, sabemos que la Comisión Gestora prepara nuevas carreras, entre ellas "Minas y petróleos" ${ }^{34}$ que contradicen frontalmente al proyecto originario, esto es, conciencializar el respeto a la Pacha Mama / Madre Tierra, como principio bioético clave que nos diferencia de las propuestas educativas pro-occidentales. Para un Ecuador con economía dependiente de la explotación petrolera que implica la destrucción de lo que queda de la selva amazónica, es imprescindible el petróleo, la modernidad lo requiere.

El principio de la territorialización de la Educación Superior, sostenida por el proyecto originario y bien ganada mediante la Sentencia No. 0008-09-SAN-CC, caso No. 0027-09-AN del 9 de diciembre del 2009, que implica la construcción institucional y académica de la Universidad a partir de los territorios de los pueblos y nacionalidades y no como creación de simples extensiones, ha sido anulada e invisibilizada una vez más por la Comisión Gestora. Sin esta institucionalidad donde jueguen un papel importante las autoridades propias y el "ayllu" 35 o comunidad, la universidad será sólo una actividad de pocos privilegiados; pero, ante todo, aislados de la realidad social - geográfica y las aspiraciones de los pueblos y nacionalidades. Será una universidad creada desde y para la ciudad. Está claro entonces la vigencia de la lógica colonial, de la lucha interminable entre la civilización y la barbarie para favorecer al capitalismo.

Estos entre otros, son hechos que emergen del patrón de poder colonial vigente en el Ecuador y en la mayoría de Estados Nación coloniales aún. No son hechos aislados o casuales, son acontecimientos que deben suceder como si fueran previamente programados y con todas las implicancias que guarda el concepto de la "colonialidad del poder", es decir, donde concurren el racismo y la urgencia de que el capitalismo contemporáneo de una vez por todas arrase con todo lo diferente, lo diverso, lo anticuado, es decir, lo indio.

Los acontecimientos actuales de la Universidad Intercultural Amawtay Wasi, a partir del llamado proceso de restitución, con la complicidad consciente o inconsciente de la dirigencia de la CONAIE y con el papel determinante de los "indígenas funcionales", han conseguido una vergonzosa regresión tanto como proyecto educativo y como derechos humanos y constitucionales hasta ahora ganados. El proyecto originario de la Universidad ha dado un giro inesperado de 360 grados para ser un proyecto de universidad convencional, de universidad occidentalizada con nombre de intercultural. El proyecto de la Universidad Intercultural de las Nacionalidades y Pueblos Indígenas "Amawtay Wasi", es un proyecto atrapado en los tentáculos de la "colonialidad del poder".

\section{Conclusiones}

- El proyecto originario de la Universidad Intercultural de las Nacionalidades y Pueblos Indígenas "Amawtay Wasi", que surge a finales de la década de los 9o, cuando aún el movimiento indígena era un

\footnotetext{
${ }^{34}$ Ver noticia de diario El Universo, que fue publicado el 17 de febrero del 2019. Disponible en: https://www.eluniverso.com/noticias/2019/02/17/nota/7192169/ comision-u-amawtay-wasi-alista-reapertura.

35 Es más que una simple familia constituida por padres e hijos y otros parentescos cercanos. Es una familia cósmica en plena relación entre todos los seres que lo cohabitan. Es un espacio dentro de la madre tierra donde se encuentran las plantas, los animales, los minerales, el agua, el fuego, el aire y la tierra. Pero además se encuentra presente la dimensión espiritual, los sitios sagrados, las wakas, los espíritus de nuestros antepasados, las energías de los otros seres, el gran espíritu. Es un verdadero tejido social, político, económico y espiritual.
} 
movimiento emergente en el Ecuador, plantea un proyecto de educación superior alternativo, que surge desde la inspiración filosófica de los pueblos originarios de Abya Yala, que pretende recuperar, sistematizar y criar saberes y conocimientos, negados e invisibilizados por la colonia y el "Moderno Estado Nación”" ${ }^{36}$ y que ofertaba su gran camino educativo a todas las sociedades en pos de la construcción del estado plurinacional y la sociedad intercultural. De verdad que es un proyecto sui géneris que asusta a la colonialidad del poder.

El proyecto planteaba y plantea una ruptura epistémica no sólo en la estructura de la tradición institucional universitaria, sino especialmente en su episteme o epistemes, tomando como ejes fundamentales para la construcción del conocimiento e investigación el Yachay ${ }^{37}$ - Ruray ${ }^{38}$, y el Ushay ${ }^{39}$ - Munay ${ }^{40}$ confluyendo todos en el Kawsay ${ }^{41} \mathrm{o}$ Vida, la pluralidad como abundancia y riqueza, la relacionalidad como tejido infinito de conocimientos y el biocentrismo como aglutinador de todo lo que existe a nivel energético y espiritual.

- La suspensión definitiva de la Universidad Intercultural de las Nacionalidades y Pueblos Indígenas "Amawtay Wasi", constituye la manifestación más clara o la materialización práctica de la segregación racial vigente en nuestros países, institución que vive disimuladamente en la vida cotidiana de quienes dirigen el "Moderno Estado Nación" y en una sociedad organizada en clases, como no puede ser de otra manera dentro de un régimen capitalista moderno.

Era necesario aplicar el oculto "Apertheid Epistémico" en este caso, pues la colonialidad, no puede admitir aberraciones a sus leyes que rigen la construcción de conocimientos desde el punto de vista occidental. Todo se encuentra debidamente fundamentado y organizado. El conocimiento, siendo el sustento principal de la ciencia y la tecnología modernas, debe obedecer al orden epistémico establecido so pena de ser invalidado y calificado de no científico.

- A los Estados coloniales les gusta mucho hablar de inclusión como si esto fuera un gran favor para los diferentes. No tienen tapujos para decirnos que la homogenización es el requisito para la modernización del capitalismo. Por ello reparten dádivas como becas y cupos para jóvenes indígenas y afrodescendientes para el ingreso en sus universidades cuyo gran fruto a la postre es la enajenación cultural civilizatoria.

Constituir o fundar universidades interculturales por parte de los Estados coloniales tampoco es problema, siempre que estos en el fondo sigan siendo "universidades occidentalizadas", aunque, en la forma se llamen interculturales. La mayoría de las universidades interculturales en México son un buen ejemplo. Los Estados coloniales han sido capaces de todo, de hacer reformas agrarias, de implementar grandes proyectos de desarrollo, de lanzar programas de educación intercultural bilingüe y porqué no de fundar universidades interculturales? El pequeño gran detalle está en que la colonialidad del poder nunca les permitirá salirse del paradigma civilizatorio de occidente en sus variantes de izquierda o de derecha, del euronorteamericanismo, del orden epistémico mundialmente establecido, pero siempre en la vergonzosa posición de subdesarrollados, mirando de rodillas a los desarrollados en el imposible sueño de ser como ellos.

\section{Lista de referencias}

CES (2013). Resolución RPC-SE-NO. 049-2013 de 4 de noviembre del 2013, mediante el cual se resuelve la Suspensión Definitiva de la Universidad Intercultural Amawtay Wasi. Quito. Recuperado el 30 de agosto del 2019, de: https://www.google.com.ec/url?sa=t\&rct=j\&q=\&esrc=s\&source=web\&cd=2\&ved=2a hUKEwjkmZ7t6a3kAhUGR6wKHWNADdgQFjABegQIAhAC\&url=http\%3A\%2F\%2Fwww.ces.gob.

\footnotetext{
${ }^{36}$ Este es el nombre creado por el pensador peruano “Aníbal Quijano" para estudiar y caracterizar al proyecto de estado implantado en América Latina y que hasta hoy se encuentra inconcluso.

37 Saber.

${ }^{38}$ Hacer.

39 Poder.

${ }^{40}$ Trascender.

${ }^{41}$ Vida.
} 
ec\%2Findex.php\%3Foption\%3Dcom_sobipro\%26task\%3Ddownload.file\%26fid\%3D231.1854\%26sid \%3D741\%26Itemid\%3Do\&usg=AOvVawoOp9XnCLvw-iiqBd85nf3v

Corte Constitucional, (2009). Sentencia No. 0008-09-SAN-CC. Caso No. 0027-09-AN. Acción constitucional por Incumplimiento propuesto por la Universidad Intercultural de las Nacionalidades y Pueblos Indígenas "Amawtay Wasi" en contra del Consejo Nacional de Educación Superior CONESUP. Quito. Recuperado el 30 de agosto del 2019, de: https://www.google.com.ec/url?sa=t\&rct=j\&q=\&esrc=s\&source=web \&cd=1\&ved=2ahUKEwir1rmn4K3kAhVomKoKHZmfD34QFjAAegQIABAC\&url=http\%3A\%2F\%2Fw ww.advaserver.com\%2Fa2\%2Fobjetos\%2Fadjunto.cfm\%3Fcodcontenido\%3D1099\%26codcampo\%3 D21\%26aplicacion\%3Dappoo3\%26cnl\%3D3\%26opc\%3D4\&usg=AOvVaw3pYKo25BVl_CEMg-qhXtv

Grosfoguel, R. (2013). Racismo/sexismo epistémico, universidades occidentalizadas y los cuatro genocidios/ epistemicidios del largo siglo XVI. University of California, Berkeley, USA.

Quijano, A. (2007). Colonialidad del Poder y Clasificación Social, en S. Castro-Gómez y R. Grosfoguel (Eds.) El Giro Decolonial: Reflexiones para una diversidad epistémica más allá del capitalismo global. Pontificia Universidad Javeriana / Siglo del Hombre Editores, Bogotá.

Rabaka, R. (2010). Against Epistemic Apartheid: W.E.B. Du Bois and the Disciplinary Decadence of Sociology. Lanham, MD: Rowman \& Littlefield Publishers.

RUIICAY (2018). El camino de la Ruiicay. "La decolonización del pensamiento, la razón y el ser en Abya Yala". Universidaddelas Regiones Autónomas dela Costa Caribe NicaraguenseURACCAN. Managua. Recuperado el 30 de agosto del 2019, de: http://www.uraccan.edu.ni/institucionalfile/el-camino-de-la-ruiicay

UINPI AW. (2012). Aprender en la Sabiduría y el Buen Vivir / Sumak Yachaypi Alli Kawsaypipash Yachaikuna / Learning Wisdom and the Good Way to Live. Universidad Intercultural Amawtay Wasi. Colección Amawta Runakunapak Yachay ARI No. 1. Segunda Edición. Quito, Ecuador: Graficas Amza. 